\title{
Qualidade de vida em cuidadores de crianças e adolescentes com transtorno do espectro autista: estudo comparativo entre sexos
}

\author{
MIRELLA FIUZA LOSAPIO \\ Universidade de São Paulo (USP), Ribeirão Preto, SP, Brasil. \\ E-mail:mflosapio@hcrp.usp.br \\ ERIKSON FELIPE FURTADO \\ Universidade de São Paulo (USP), Ribeirão Preto, SP, Brasil. \\ E-mail: efurtado@fmrp.usp.br
}

\section{Resumo}

O transtorno do espectro autista (TEA) é uma alteração do neurodesenvolvimento que frequentemente se apresenta com prejuízos sociais e dificuldades de aprendizagem e de autonomia, e impacta diretamente a família. Consequentemente, os cuidadores de crianças e adolescentes com esse diagnóstico precisam lidar com maiores níveis de estresse, que levam a baixos índices de qualidade de vida. Na literatura há descrição de que as meninas com TEA apresentam-se mais comprometidas que os meninos. Este estudo teve como objetivos avaliar a qualidade de vida em cuidadores de crianças e adolescentes com TEA e realizar comparação deste constructo entre cuidadores de pacientes do sexo feminino e masculino. Realizou-se uma entrevista semiestruturada e aplicou-se o instrumento WHOQOL-bref em 62 cuidadores de crianças ou adolescentes atendidos no ambulatório de TEA de um hospital-escola de Ribeirão Preto. A maioria da amostra era composta por mães (85\%), indivíduos "não brancos", com ensino médio completo e renda familiar abaixo de dois salários mínimos. Houve predomínio de qualidade de vida mais baixa nos cuidadores de crianças e adolescentes do sexo feminino, em comparação com cuidadores do sexo masculino, porém sem relevância estatística. Contudo, nas questões relacionadas às condições socioeconômicas, houve associação com relevância estatística, de modo que os cuidadores de pacientes do sexo feminino pontuavam com menores escores nessa área. Este estudo não encontrou associação entre qualidade de vida nos cuidadores e sexo das crianças e adolescentes com TEA. 


\section{Palavras-chave}

Transtorno do espectro autista. Transtorno autístico. Qualidade de vida. Cuidadores. Sexo.

\section{INTRODUÇÃO}

O diagnóstico de transtorno do espectro autista (TEA) sofreu diversas alterações de critérios, classificações e nomenclatura desde 1980, ano do lançamento da terceira edição do Manual diagnóstico e estatístico de transtornos mentais (DSM), na qual o autismo foi reconhecido pela primeira vez como uma condição específica. Já em 2013, o DSM, em sua quinta versão, estabeleceu a expressão atual e a classificação dimensional desse transtorno.

Em relação aos critérios diagnósticos, a classificação atual exige déficits na comunicação e interação social e um padrão de comportamentos ou interesses restritos e repetitivos. O TEA é definido como uma alteração do neurodesenvolvimento, e, por isso, as características dele devem se apresentar precocemente (AMERICAN PSYCHIATRIC ASSOCIATION, 2013). O DSM-5 define ainda os seguintes especificadores do diagnóstico de TEA: presença ou ausência de linguagem verbal e de deficiência intelectual, associação com alguma condição médica ou genética ou ambiental e comorbidades psiquiátricas. Há também a possibilidade de classificação do diagnóstico em níveis de gravidade (AMERICAN PSYCHIATRIC ASSOCIATION, 2013). Dessa forma, crianças e adolescentes com TEA apresentam ampla variedade de prejuízos, desde déficits sociais até dificuldades de aprendizagem e de autonomia, o que impacta diretamente a família.

Diversas patologias crônicas desencadeiam impactos para as famílias dos pacientes, e, consequentemente, o conceito de estresse do cuidador tem sido estudado em diversas áreas da saúde (CHIAO; WU; HSIAO, 2015; GENG et al., 2018; BEKTAS et al., 2020). Segundo Fávero e Santos (2005), as crianças e os adolescentes com TEA apresentam demandas novas para a família, que geram necessidade de adaptação às mudanças advindas dessa excepcionalidade e, dessa forma, sobrecarga nos familiares, desencadeando estresse nos cuidadores. Além de mudanças, frequentemente os cuidadores apresentam preocupações em relação aos déficits característicos do TEA, gerando ainda mais estresse (KOEGEL et al., 1992). Uma revisão de literatura realizada em 2016 constatou que fatores do paciente, como gravidade do TEA, sintomas ansiosos e depressivos no cuidador e condição socioeconômica da família, são variáveis que in- 
terferem na qualidade de vida de familiares de crianças com esse transtorno (MIELE; AMATO, 2016). Autores identificaram que altos níveis de estresse nos cuidadores implicam redução da qualidade de vida (BONIS, 2016; TEN HOOPEN et al., 2020; BARROS et al., 2019; MARSACK-TOPOLEWSKI; CHURCH, 2019; MARSACK; SAMUEL, 2017; HOEFMAN et al., 2014).

A Organização Mundial da Saúde (OMS) define qualidade de vida como "a percepção do indivíduo de sua posição na vida, no contexto da cultura e dos sistemas de valores em que vive e em relação aos seus objetivos, expectativas, padrões e preocupações" (THE WHOQOL GROUP, 1998, p. 551-558). A autopercepção de reduzida qualidade de vida entre cuidadores afeta o estilo de cuidado parental, o manejo dos comportamentos desafiadores da criança, o funcionamento familiar e o relacionamento conjugal (BONIS, 2016).

O diagnóstico de TEA tem aumentado nos últimos anos. A mais recente análise epidemiológica realizada nos Estados Unidos, em 2020, detectou taxa de prevalência de aproximadamente um caso para cada grupo de 54 indivíduos, ou 18,5 casos por mil, para a faixa etária de 8 anos (MAENNER et al., 2020).

Diante da elevada prevalência desse diagnóstico associada à ampla gama de prejuízos, é relevante o estudo da qualidade de vida do cuidador dessas crianças e desses adolescentes.

Dados da literatura evidenciam que a apresentação clínica do TEA é mais sutil em meninas, e, por isso, muitas delas com quadros leves são subdiagnosticadas, gerando um predomínio de casos graves entre pacientes do sexo feminino que estão em seguimento psiquiátrico por TEA (BARON-COHEN et al., 2009; KOPP; BECKUNG; GILLBERG, 2010; KOTHARI et al., 2013). Ser cuidador de um jovem com maior gravidade gera mais demandas e maior preocupação, impactando diretamente a qualidade de vida dos familiares. Esse é o argumento norteador da comparação da qualidade de vida, nos cuidadores, por sexo dos pacientes.

Dessa forma, este estudo teve como objetivos avaliar a qualidade de vida dos cuidadores de crianças e adolescentes com diagnóstico de TEA e compará-la em cuidadores de pacientes do sexo feminino com os do sexo masculino, em seguimento ambulatorial em um hospital universitário da cidade de Ribeirão Preto.

\section{METODOLOGIA}

Trata-se de estudo observacional, transversal, aberto, não cego, de casuística clínica, realizado no Serviço de Psiquiatria da Infância e Adolescência 
(SPQIA) do Hospital das Clínicas da Faculdade de Medicina de Ribeirão Preto, Universidade de São Paulo (HC-FMRP-USP). Os dados aqui descritos foram coletados do Ambulatório de Transtorno do Espectro Autista e Deficiência Intelectual (PQITEADI), pertencente ao SPQIA.

A pesquisa foi aprovada pelo Comitê de Ética e Pesquisa do HC-FMRP-USP, por meio do Processo HCRP n. 2.911.406.

O estudo foi composto por responsáveis de 62 crianças e adolescentes, que os acompanhavam em consulta psiquiátrica no ambulatório citado. Os pacientes apresentavam idade entre 3 e 17 anos, já estavam em seguimento clínico nesse ambulatório e já possuíam diagnóstico de TEA, segundo os critérios diagnósticos vigentes no DSM-5 (AMERICAN PSYCHIATRIC ASSOCIATION, 2013).

Logo após a consulta médica no ambulatório citado, os responsáveis foram convidados a participar da pesquisa e, depois do aceite, direcionados para um consultório no mesmo prédio onde acontece o ambulatório. Então, os responsáveis legais foram instruídos a ler e assinar o Termo de Consentimento Livre e Esclarecido. O critério de inclusão foi residir com criança ou adolescente que apresente diagnóstico clínico de TEA, como diagnóstico principal, realizado conforme os procedimentos clínicos vigentes no SPQIA do HC-FMRP-USP, em acompanhamento contínuo de pelo menos seis meses de duração. Os critérios de exclusão foram não concordância em assinar o Termo de Consentimento Livre e Esclarecido, ser responsável por crianças ou adolescentes cujo diagnóstico principal era deficiência intelectual e não residir com o paciente.

Após o recrutamento descrito anteriormente, realizou-se entrevista semiestruturada com os participantes, com o objetivo de obter dados sociodemográficos e clínicos das crianças e dos adolescentes. Aplicou-se ainda o instrumento World Health Organization Quality of Life-bref (WHOQOL-bref). Esse instrumento é uma simplificação do questionário WHOQOL-100, desenvolvido pela OMS, com o objetivo de explorar qualidade de vida. O WHOQOL-bref utilizado neste estudo é composto por 26 questões, sendo duas questões gerais e outras 24 que avaliam os seguintes domínios: físico, psicológico, relações sociais e meio ambiente (THE WHOQOL GROUP, 1998). As questões foram lidas pela entrevistadora e pontuadas pelo responsável da criança, com gradação entre 1 e 5 para cada alternativa, de acordo com a frequência que percebe cada uma das questões em sua vida.

Ao final da coleta, os dados foram submetidos à estatística descritiva e inferencial. Obtiveram-se os dados estatísticos com a utilização do programa 
de estatística Statistical Package for Social Science, a partir de um banco de dados do mesmo programa. Utilizaram-se estatísticas descritivas para caracterizar a amostra. Para análise de inferência, adotaram-se testes estatísticos adequados ao padrão de distribuição das variáveis. Para a comparação das variáveis contínuas e a comparação de médias, foram realizadas análises de variância (ANOVA). Para diferenças entre proporções, utilizou-se o teste qui-quadrado. O nível de significância utilizado foi de 5\% para todos os testes. Fez-se testagem estatística com emprego de técnicas não paramétricas, quando necessário, em função do tamanho dos grupos ou da distribuição das variáveis.

Os artigos aqui citados foram selecionados com base em ano de publicação e idioma, com o objetivo de trazer para este estudo dados relevantes e atuais encontrados por outros autores. Os idiomas escolhidos foram português, inglês e espanhol, por limitações dos autores em relação a outros idiomas.

\section{RESULTADOS}

Participaram da pesquisa 62 familiares de crianças ou adolescentes atendidos no PQITEADI. Os participantes apresentavam idades entre 24 e 63 anos. A Tabela 1 sintetiza os dados sociodemográficos dos cuidadores.

Tabela 1 Caracterização da amostra (dos cuidadores)

\begin{tabular}{cccc}
\hline Variáveis & Categorias & N & \% \\
\hline \multirow{2}{*}{ Sexo } & Masculino & 6 & 10 \\
& Feminino & 56 & 90 \\
\hline \multirow{3}{*}{ Cor da pele } & Branca & 29 & 47 \\
& Parda & 29 & 47 \\
& Negra & 4 & 6 \\
\hline \multirow{3}{*}{ Parentesco } & Mãe & 53 & 85 \\
& Pai & 6 & 10 \\
Escolaridade & Avó & 3 & 5 \\
\hline \multirow{3}{*}{ Renda familiar } & Ensino fundamental & 19 & 31 \\
& Ensino médio & 30 & 48 \\
& Ensino superior & 13 & 44 \\
\hline & Abaixo de 2 SM & 27 & 35 \\
\hline
\end{tabular}

SM - salário mínimo; $\mathrm{N}$ - número de sujeitos; \% - porcentagem.

Fonte: Elaborada pelos autores. 
Entre os cuidadores, houve predomínio de mães, correspondendo a 85\% do total de participantes. Além de mãe e pai, alguns participantes eram avós das crianças ou dos adolescentes atendidos, perfazendo um total de $5 \%$.

A seguir, a Tabela 2 apresenta a descrição das características sociodemográficas, por meio de caracterização do sexo dos pacientes do ambulatório e informações do cuidador, como: cor da pele, renda familiar e escolaridade. Observa-se que a cor da pele teve distribuição semelhante entre ambos os sexos, com discreto predomínio de "não brancos" (cores parda ou preta), porém sem relevância estatística. A variável renda familiar também ficou bem distribuída entre os sexos. As diferenças encontradas não foram estatisticamente significantes na amostra. A única variável que se comportou com distribuição estatisticamente significante entre os sexos foi a escolaridade do cuidador. Observa-se predomínio de cuidadores mais bem instruídos em pacientes do sexo masculino $(\mathrm{p}=0,016)$.

Tabela 2 Caracterização sociodemográfica dos cuidadores, conforme distribuição por sexo das crianças e dos adolescentes

\begin{tabular}{|c|c|c|c|c|}
\hline Variáveis & $\begin{array}{c}\text { Masculino } \\
\text { (N=42; } \\
67,7 \%)\end{array}$ & $\begin{array}{c}\text { Feminino } \\
(\mathrm{N}=20 \\
32,3 \%)\end{array}$ & $\begin{array}{c}\text { Total } \\
(\mathrm{N}=62 ; \\
100 \%)\end{array}$ & $\chi^{2}(\mathrm{df} ; p)$ \\
\hline \multicolumn{5}{|l|}{ Cor da pele } \\
\hline Parda ou preta & $20(32,2 \%)$ & $13(21,0 \%)$ & $33(53,2 \%)$ & 1,64 (1; n.s.) \\
\hline Branca & $22(35,5 \%)$ & $7(11,3 \%)$ & $29(46,8 \%)$ & \\
\hline \multicolumn{5}{|l|}{ Renda familiar } \\
\hline Abaixo de 2 SM & $18(29,0 \%)$ & $9(14,5 \%)$ & $27(43,5 \%)$ & 0,68 (2; n.s.) \\
\hline Entre 2 e 4 SM & $14(22,6 \%)$ & $8(12,9 \%)$ & $22(35,5 \%)$ & \\
\hline Acima de 4 SM & $10(16,2 \%)$ & $3(4,8 \%)$ & $13(21,0 \%)$ & \\
\hline \multicolumn{5}{|l|}{ Escolaridade } \\
\hline Ensino fundamental & $8(12,9 \%)$ & $11(17,7 \%)$ & $19(30,6 \%)$ & $8,28(2 ; 0,016)^{*}$ \\
\hline Ensino médio & $24(38,7 \%)$ & $6(9,7 \%)$ & $30(48,4 \%)$ & \\
\hline Ensino superior & $10(16,2 \%)$ & $3(4,8 \%)$ & $13(21,0 \%)$ & \\
\hline
\end{tabular}

$\mathrm{N}$ - número de sujeitos; \% - porcentagem; $\mathrm{X}^{2}$ - coeficiente do qui-quadrado de Pearson; $\mathrm{df}$ - grau de liberdade (degree offreedom); $p$ - nível de significância estatística; n.s. - não significante (abaixo de 0,05).

Fonte: Elaborada pelos autores. 
Foi realizada análise das pontuações do WHOQOL-bref por domínio do instrumento, em cada um dos sexos dos pacientes. Os resultados estão apresentados na Tabela 3. A observação dessa tabela permite a inferência de que, embora não tenha sido observada diferença estatisticamente significante na análise dos domínios, há um padrão em todos os domínios, de escores menores quando se trata de cuidadores de pacientes do sexo feminino, o que significa que os cuidadores das meninas pontuam para uma menor qualidade de vida em comparação aos cuidadores das crianças e dos adolescentes do sexo masculino.

Tabela 3 Domínios de qualidade de vida dos cuidadores, conforme sexo do paciente

\begin{tabular}{|c|c|c|c|c|}
\hline & \multicolumn{2}{|c|}{ Grupos por sexo [M (DP)] } & \multirow{2}{*}{$\begin{array}{c}\text { Total } \\
(\mathrm{N}=62 ; 100 \%)\end{array}$} & \multirow{2}{*}{$\begin{array}{l}\text { ANOVA } \\
(\mathrm{Z} ; \mathrm{df} ; p)\end{array}$} \\
\hline & $\begin{array}{c}\text { Masculino } \\
(\mathrm{N}=42 ; 67,7 \%)\end{array}$ & $\begin{array}{c}\text { Feminino } \\
(\mathrm{N}=20 ; 32,3 \%)\end{array}$ & & \\
\hline $\begin{array}{l}\text { Capacidade } \\
\text { física }\end{array}$ & $14,45(2,54)$ & $13,31(3,30)$ & $14,08(2,83)$ & 2,$22 ; 1 ; 0,14$ (n.s.) \\
\hline $\begin{array}{l}\text { Bem-estar } \\
\text { psicológico }\end{array}$ & $14,29(2,65)$ & $14,00(3,22)$ & $14,19(2,82)$ & 0,$14 ; 1 ; 0,71$ (n.s.) \\
\hline Relações sociais & $15,08(3,07)$ & $14,53(3,40)$ & $14,90(3,16)$ & $\begin{array}{c}0,40 ; 1 ; 0,53 \\
\text { (n.s.) }\end{array}$ \\
\hline Meio ambiente & $14,06(2,23)$ & $13,13(2,37)$ & $13,76(2,30)$ & $\begin{array}{l}\text { 2,29; } 1 ; 0,14 \\
\quad \text { (n.s.) }\end{array}$ \\
\hline Avaliação geral & $13,57(2,48)$ & $12,40(3,02)$ & $13,19(2,70)$ & 2,$62 ; 1 ; 0,11$ (n.s.) \\
\hline
\end{tabular}

M - média aritmética; DP - desvio padrão; $\mathrm{N}$ - número de sujeitos; \% - porcentagem; $\mathrm{Z}$ - coeficiente do teste de diferença de médias; df - grau de liberdade (degree of freedom); $p$ - nível de significância; n.s. - não significante.

Fonte: Elaborada pelos autores.

O padrão de escores menores no WHOQOL-bref em cuidadores de pacientes do sexo feminino fica ainda mais evidente na avaliação separada das questões. Há, inclusive, três questões do instrumento cujas diferenças entre as médias dos escores são estatisticamente significantes. As questões citadas são as referentes a ter dinheiro suficiente, acesso a informações e capacidade de locomoção. $\mathrm{O}$ valor de $p$ é 0,02 nas três questões, como se observa na Tabela 4. 


\begin{tabular}{|c|c|c|c|c|c|c|}
\hline & & \multicolumn{3}{|c|}{$\begin{array}{c}\text { Intervalo de } \\
\text { confiança (95\%) }\end{array}$} & \multirow[b]{2}{*}{ Z } & \multirow[b]{2}{*}{$p$} \\
\hline & & M & $\begin{array}{l}\text { Lim. } \\
\text { inf. }\end{array}$ & $\begin{array}{l}\text { Lim. } \\
\text { sup. }\end{array}$ & & \\
\hline \multirow{3}{*}{$\begin{array}{l}\text { Como você avaliaria sua qualidade } \\
\text { de vida? }\end{array}$} & Masc & 3,24 & 3,02 & 3,45 & 0,79 & 0,38 \\
\hline & Fem & 3,05 & 2,61 & 3,49 & & \\
\hline & Total & 3,18 & 2,98 & 3,38 & & \\
\hline \multirow{3}{*}{$\begin{array}{l}\text { Quão satisfeito(a) você está com a } \\
\text { sua saúde? }\end{array}$} & Masc & 3,55 & 3,28 & 3,82 & 2,99 & 0,09 \\
\hline & Fem & 3,15 & 2,77 & 3,53 & & \\
\hline & Total & 3,42 & 3,20 & 3,64 & & \\
\hline \multirow{3}{*}{$\begin{array}{l}\text { Em que medida você acha que sua dor } \\
\text { (física) o(a) impede de fazer o que } \\
\text { precisa? }\end{array}$} & Masc & 3,93 & 3,64 & 4,22 & 2,42 & 0,13 \\
\hline & Fem & 3,50 & 2,94 & 4,06 & & \\
\hline & Total & 3,79 & 3,53 & 4,05 & & \\
\hline \multirow{3}{*}{$\begin{array}{l}\text { Quanto você precisa de algum } \\
\text { tratamento médico para levar sua vida } \\
\text { diária? }\end{array}$} & Masc & 3,79 & 3,48 & 4,09 & 0,66 & 0,42 \\
\hline & Fem & 3,55 & 2,97 & 4,13 & & \\
\hline & Total & 3,71 & 3,44 & 3,98 & & \\
\hline \multirow{3}{*}{ Quanto você aproveita a vida? } & Masc & 3,00 & 2,71 & 3,29 & 0,03 & 0,86 \\
\hline & Fem & 3,05 & 2,51 & 3,59 & & \\
\hline & Total & 3,02 & 2,76 & 3,27 & & \\
\hline \multirow{3}{*}{$\begin{array}{l}\text { Em que medida você acha que a sua } \\
\text { vida tem sentido? }\end{array}$} & Masc & 4,24 & 3,98 & 4,49 & 0,15 & 0,70 \\
\hline & Fem & 4,15 & 3,74 & 4,56 & & \\
\hline & Total & 4,21 & 4,00 & 4,42 & & \\
\hline \multirow{3}{*}{ Quanto você consegue se concentrar? } & Masc & 3,52 & 3,29 & 3,75 & 1,68 & 0,20 \\
\hline & Fem & 3,25 & 2,85 & 3,65 & & \\
\hline & Total & 3,44 & 3,24 & 3,63 & & \\
\hline
\end{tabular}




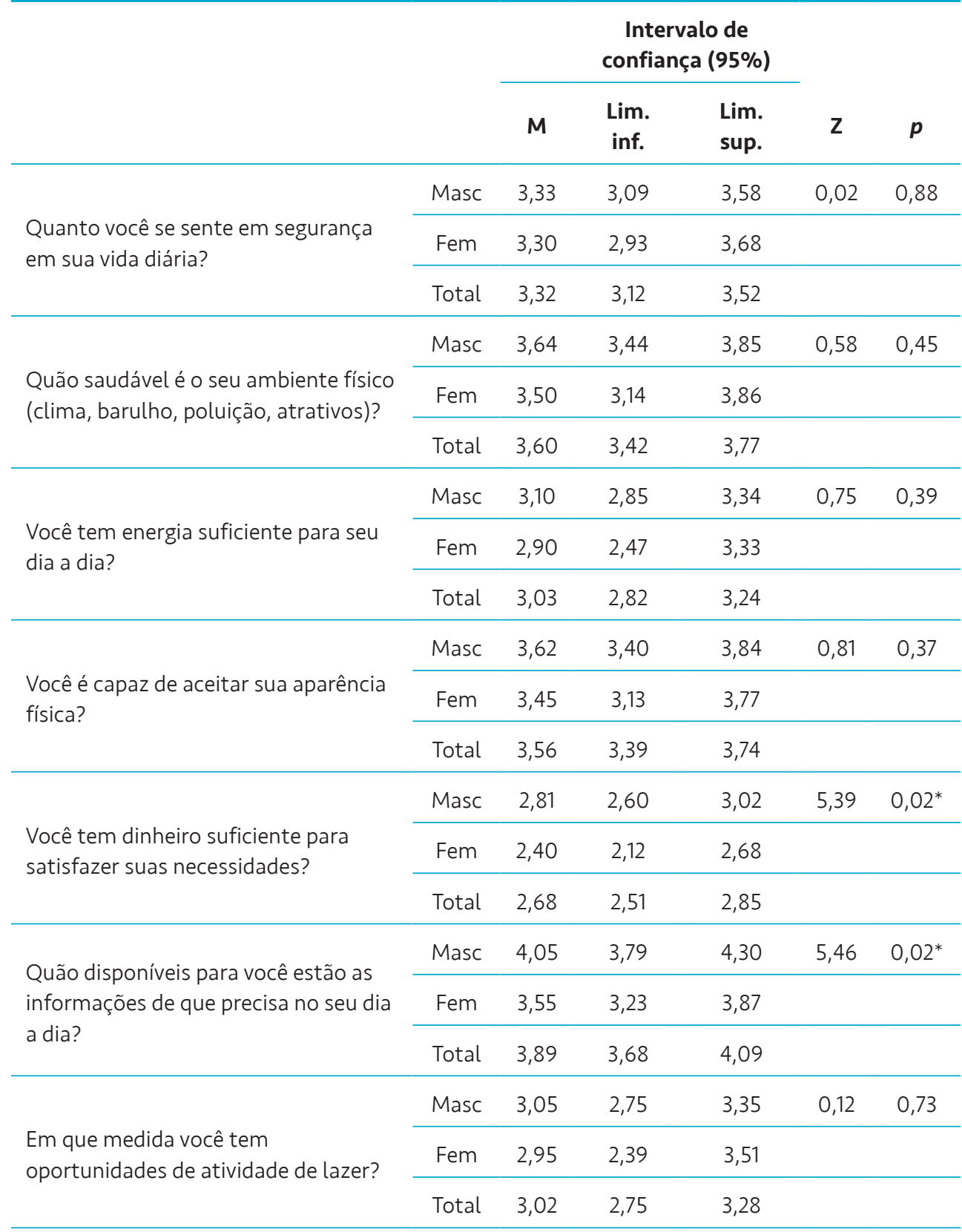




\begin{tabular}{|c|c|c|c|c|c|c|}
\hline & & \multicolumn{3}{|c|}{$\begin{array}{c}\text { Intervalo de } \\
\text { confiança }(95 \%)\end{array}$} & \multirow[b]{2}{*}{ z } & \multirow[b]{2}{*}{$p$} \\
\hline & & $M$ & $\begin{array}{l}\text { Lim. } \\
\text { inf. }\end{array}$ & $\begin{array}{l}\text { Lim. } \\
\text { sup. }\end{array}$ & & \\
\hline \multirow{3}{*}{$\begin{array}{l}\text { Quão bem você é capaz de se } \\
\text { locomover? }\end{array}$} & Masc & 4,36 & 4,18 & 4,54 & 5,77 & $0,02^{*}$ \\
\hline & Fem & 3,90 & 3,47 & 4,33 & & \\
\hline & Total & 4,21 & 4,03 & 4,39 & & \\
\hline \multirow{3}{*}{$\begin{array}{l}\text { Quão satisfeito(a) você está com o } \\
\text { seu sono? }\end{array}$} & Masc & 3,26 & 2,97 & 3,55 & 1,55 & 0,22 \\
\hline & Fem & 2,95 & 2,53 & 3,37 & & \\
\hline & Total & 3,16 & 2,93 & 3,40 & & \\
\hline \multirow{3}{*}{$\begin{array}{l}\text { Quão satisfeito(a) você está com sua } \\
\text { capacidade de desempenhar as } \\
\text { atividades do seu dia a dia? }\end{array}$} & Masc & 3,60 & 3,35 & 3,84 & 0,42 & 0,52 \\
\hline & Fem & 3,45 & 3,03 & 3,87 & & \\
\hline & Total & 3,55 & 3,34 & 3,76 & & \\
\hline \multirow{3}{*}{$\begin{array}{l}\text { Quão satisfeito(a) você está com sua } \\
\text { capacidade para o trabalho? }\end{array}$} & Masc & 3,26 & 2,98 & 3,55 & 0,66 & 0,42 \\
\hline & Fem & 3,05 & 2,56 & 3,54 & & \\
\hline & Total & 3,19 & 2,95 & 3,44 & & \\
\hline \multirow{3}{*}{$\begin{array}{l}\text { Quão satisfeito(a) você está consigo } \\
\text { mesmo(a)? }\end{array}$} & Masc & 3,74 & 3,52 & 3,96 & 1,06 & 0,31 \\
\hline & Fem & 3,55 & 3,27 & 3,83 & & \\
\hline & Total & 3,68 & 3,51 & 3,85 & & \\
\hline \multirow{3}{*}{$\begin{array}{l}\text { Quão satisfeito(a) você está com suas } \\
\text { relações pessoais (amigos, parentes, } \\
\text { conhecidos, colegas)? }\end{array}$} & Masc & 4,02 & 3,76 & 4,29 & 0,27 & 0,61 \\
\hline & Fem & 3,90 & 3,45 & 4,35 & & \\
\hline & Total & 3,98 & 3,76 & 4,21 & & \\
\hline \multirow{3}{*}{$\begin{array}{l}\text { Quão satisfeito(a) você está com sua } \\
\text { vida sexual? }\end{array}$} & Masc & 3,62 & 3,36 & 3,88 & 0,52 & 0,48 \\
\hline & Fem & 3,45 & 3,01 & 3,89 & & \\
\hline & Total & 3,56 & 3,35 & 3,78 & & \\
\hline
\end{tabular}


Tabela 4 Questões do "WHOQOL - Qualidade de Vida dos Cuidadores", conforme sexo do paciente (continuação)

\begin{tabular}{|c|c|c|c|c|c|c|}
\hline & & \multicolumn{3}{|c|}{$\begin{array}{c}\text { Intervalo de } \\
\text { confiança }(95 \%)\end{array}$} & \multirow[b]{2}{*}{ Z } & \multirow[b]{2}{*}{$p$} \\
\hline & & $M$ & $\begin{array}{l}\text { Lim. } \\
\text { inf. }\end{array}$ & $\begin{array}{l}\text { Lim. } \\
\text { sup. }\end{array}$ & & \\
\hline \multirow{3}{*}{$\begin{array}{l}\text { Quão satisfeito(a) você está com o } \\
\text { apoio que você recebe de seus } \\
\text { amigos? }\end{array}$} & Masc & 3,67 & 3,36 & 3,97 & 0,20 & 0,66 \\
\hline & Fem & 3,55 & 3,11 & 3,99 & & \\
\hline & Total & 3,63 & 3,38 & 3,87 & & \\
\hline \multirow{3}{*}{$\begin{array}{l}\text { Quão satisfeito(a) você está com as } \\
\text { condições do local onde mora? }\end{array}$} & Masc & 3,62 & 3,36 & 3,88 & 2,06 & 0,16 \\
\hline & Fem & 3,30 & 2,93 & 3,68 & & \\
\hline & Total & 3,52 & 3,31 & 3,73 & & \\
\hline \multirow{3}{*}{$\begin{array}{l}\text { Quão satisfeito(a) você está com o } \\
\text { seu acesso aos serviços de saúde? }\end{array}$} & Masc & 3,64 & 3,39 & 3,90 & 0,37 & 0,54 \\
\hline & Fem & 3,50 & 3,06 & 3,94 & & \\
\hline & Total & 3,60 & 3,38 & 3,81 & & \\
\hline \multirow{3}{*}{$\begin{array}{l}\text { Quão satisfeito(a) você está com o } \\
\text { seu meio de transporte? }\end{array}$} & Masc & 3,98 & 3,73 & 4,22 & 1,13 & 0,29 \\
\hline & Fem & 3,75 & 3,38 & 4,12 & & \\
\hline & Total & 3,90 & 3,70 & 4,10 & & \\
\hline \multirow{3}{*}{$\begin{array}{l}\text { Com que frequência você tem } \\
\text { sentimentos negativos, tais como } \\
\text { mau humor, desespero, ansiedade, } \\
\text { depressão? }\end{array}$} & Masc & 3,48 & 3,14 & 3,82 & 0,15 & 0,70 \\
\hline & Fem & 3,60 & 2,97 & 4,23 & & \\
\hline & Total & 3,52 & 3,22 & 3,81 & & \\
\hline
\end{tabular}

$M$ - média aritmética; masc - sexo masculino ( $N=42)$; fem - sexo feminino $(N=20)$; Total - amostra total $(N=62)$; $Z$ - coeficiente do teste de diferença de médias; $p$ - nível de significância; ${ }^{*}$ - estatisticamente significante $(\leq 0,05)$.

Fonte: Elaborada pelos autores.

\section{DISCUSSÃO}

Esta pesquisa permite a compreensão do quanto a sobrecarga com os cuidados de uma criança ou um adolescente com TEA impacta a qualidade de vida de seus cuidadores. Tais dados evidenciam como os familiares deste estudo relatam sentir-se quando cuidam da criança ou do adolescente com TEA e constituem importante elemento para o planejamento de intervenções dirigidas aos jovens com TEA e seus familiares. 
Observou-se que a maioria (90\%) dos acompanhantes das crianças e dos adolescentes nas consultas psiquiátricas era do sexo feminino; 85\% eram mães, apenas $10 \%$ eram pais e $5 \%$, avós. A maior parte dos estudos que avaliam acompanhantes de crianças e adolescentes em atendimento médico investiga os que estão internados, contudo estudos realizados ambulatorialmente também mostram predomínio das mães como acompanhantes (SAVIANI-ZEOTI; PETEAN, 2008; MISQUIATTI et al., 2015; VELHOTE; BOHOMOL; VELHOTE, 2016).

Ao caracterizar a amostra, o estudo identificou as proporções de cor da pele dos participantes. A definição de cor da pele em um país miscigenado como o Brasil não é simples. Um estudo do Instituto Brasileiro de Geografia e Estatística (IBGE), realizado em 2019, descreve a seguinte caracterização dessa variável na população brasileira, por autoclassificação: branca (42,7\%), preta $(9,4 \%)$, parda $(46,8 \%)$ e amarela ou indígena $(1,1 \%)$. Em suma, $57,3 \%$ se caracterizaram como "não brancos" (INSTITUTO BRASILEIRO DE GEOGRAFIA E ESTATÍSTICA, 2019). Já neste estudo, caracterizaram-se como "não brancos" $53,2 \%$ do total da amostra, uma proporção semelhante à do estudo nacional.

Outra característica importante para traçar o perfil sociodemográfico dos participantes do estudo é a renda familiar. Apenas $21 \%$ das famílias dispunham de renda acima de quatro salários mínimos. Percebe-se então que essa variável se apresentou com predomínio de baixa renda, abaixo de dois salários mínimos.

É relevante ressaltar que, na análise dos resultados, a escolaridade dos cuidadores apresentou diferença estatisticamente significante entre os sexos das crianças e dos adolescentes. Os pacientes do sexo masculino apresentaram cuidadores mais bem instruídos do que as meninas. Esse resultado pode ser consequência da diferença de gravidade entre os sexos. Segundo dados da literatura, as meninas costumam apresentar quadros mais graves que os meninos (WERLING; GESCHWIND, 2013; MAHENDIRAN et al., 2019), contudo talvez os cuidadores destes, por serem mais instruídos, mesmo quando diante de quadros mais brandos, procurem auxílio médico.

Este trabalho não encontrou associação entre qualidade de vida do cuidador e sexo da criança ou do adolescente com TEA. Apesar disso, em todos os domínios da escala WHOQOL-bref, as pontuações de cuidadores de meninas foram inferiores às pontuações de cuidadores de meninos, contudo sem relevância estatística. Não foram encontrados na literatura estudos que fizeram análise comparativa por sexo da criança com TEA, para refutar ou corroborar esse resultado. 
Já a análise separada por questões mostrou associação estatisticamente significante para os cuidadores de crianças ou adolescentes do sexo feminino com TEA em três delas. Essas questões se referiam a ter dinheiro suficiente, acesso a informações e capacidade de locomoção, que são temáticas diretamente relacionadas com condições socioeconômicas. Dessa forma, como relatado anteriormente nos resultados, os familiares de pacientes do sexo masculino eram mais instruídos e por isso podem ter mais acesso à informação, residir em locais com mais conforto e ter mais acesso a tratamentos de saúde, proporcionando melhores escores nessas questões da escala de qualidade de vida em relação aos cuidadores de pacientes do sexo feminino.

As limitações deste estudo são o número reduzido da amostra e o viés de gravidade, já que foi realizado em um hospital universitário que é referência para os pacientes graves de toda a região. Ser cuidador de um paciente com mais comprometimentos gera mais demandas e maior preocupação, o que pode ter resultado em índices piores de qualidade de vida.

Diante dos resultados apresentados, faz-se necessária a replicação das análises em outras amostras e em populações maiores, para que os resultados sejam extrapolados para todos os cuidadores de TEA. Contudo, diante dos dados aqui encontrados e dos estudos semelhantes avaliados, é importante investir em programas de qualidade de vida para os acompanhantes de jovens com TEA. Sugere-se que, na consulta, o psiquiatra esteja atento à qualidade de vida dos cuidadores e uma das estratégias de intervenção seja o cuidado com estes.

\section{CONCLUSÃO}

Este estudo buscou descrever a qualidade de vida em cuidadores de crianças e adolescentes em seguimento psiquiátrico em um ambulatório de TEA, em um hospital universitário, e, dessa forma, a amostra ficou caracterizada.

Outro foco do estudo foi comparar a qualidade de vida dos cuidadores de crianças e adolescentes do sexo feminino com os de jovens do sexo masculino. Constatou-se que não há associação entre o sexo dos cuidadores de crianças e adolescentes com TEA e qualidade de vida.

Dessa forma, fica disponível um estudo que caracteriza prejuízos de qualidade de vida em cuidadores de crianças e adolescentes com TEA, sem associação com o sexo da criança ou adolescente. Diante desse dado, destaca-se a importância de fomentar o desenvolvimento de programas de cuidado para os familiares de todos os portadores de TEA. 


\title{
Quality of life in caregivers of children and adolescents with autism spectrum disorder: a comparative study between sexes
}

\begin{abstract}
Autistic spectrum disorder (ASD) is a neurodevelopmental disorder that often presents with social impairments, learning and autonomy difficulties, that directly impact in the family. Consequently, caregivers of children and adolescents with this diagnosis need to deal with higher levels of stress, which lead to low levels of quality of life. There is a description in the literature that girls with ASD are more severe than boys. To evaluate the quality of life of caregivers of children and adolescents with ASD and compare it between caregivers of female and male patients. Semi-structured interviews and the application of the WHOQOL-bref instrument were performed in 62 caregivers of children or adolescents treated at the ASD outpatient clinic of a teaching hospital in Ribeirão Preto. Most of the sample consisted of mothers (85\%), "non-white" individuals, with complete high school education and family income below 2 minimum wages. There was a predominance of lower quality of life among caregivers of female children and adolescents, compared to male caregivers, but without statistical significance. However, in questions related to socioeconomic conditions, there was an association, with statistical relevance, so that the caregivers of female patients scored with lower scores in this area. This study found no association between quality of life in caregivers and sex of children and adolescents with ASD.
\end{abstract}

\section{Keywords}

Autism spectrum disorder. Autistic disorder. Quality of life. Caregivers. Sex.

\section{Calidad de vida en cuidadores de niños y adolescentes com trastorno del espectro autista: un estudio comparativo entre sexos}

\section{Resumen}

El trastorno del espectro autista (TEA) es un trastorno del neurodesarrollo que a menudo se presenta con deficiencias sociales, dificultades de aprendizaje y autonomía, que impacta directamente en la familia. En consecuencia, los cuidadores de niños y adolescentes con este diagnóstico deben lidiar 
con niveles más altos de estrés, que conducen a niveles bajos de calidad de vida. Hay una descripción en la literatura de que las niñas con TEA están más comprometidas que los niños. Evaluar la calidad de vida de los cuidadores de niños y adolescentes con TEA y compararla entre cuidadores de pacientes femeninos y masculinos. Se realizaron entrevistas semiestructuradas y la aplicación del instrumento WHOQOL-bref a 62 cuidadores de niños o adolescentes atendidos en el ambulatorio TEA de un hospital universitario de Ribeirão Preto. La mayor parte de la muestra estuvo conformada por madres (85\%), individuos "no blancos", con educación secundaria completa e ingresos familiares por debajo de 2 salarios mínimos. Hubo un predominio de menor calidad de vida entre los cuidadores de niñas y adolescentes, en comparación con los cuidadores masculinos, pero sin significación estadística. Sin embargo, en las preguntas relacionadas con las condiciones socioeconómicas, hubo una asociación, con relevancia estadística, por lo que los cuidadores de pacientes del sexo femenino puntuaron con puntuaciones más bajas en esta área. Este estudio no encontró asociación entre la calidad de vida de los cuidadores y el sexo de niños y adolescentes con TEA.

\section{Palabras clave}

Trastorno del espectro autista. Transtorno autístico. Calidad de vida. Cuidadores. Sexo.

\section{REFERÊNCIAS}

AMERICAN PSYCHIATRIC ASSOCIATION. Diagnostic and statistical manual of mental disorders. 5. ed. Arlington, VA: American Psychiatric Association, 2013.

BARON-COHEN, S. et al. Prevalence of autism-spectrum conditions: UK school-based population study. British Journal Psychiatry, v. 194, n. 6, p. 500-509, 2009. DOI: 10.1192/bjp.bp.108.059345

BARROS, A. L. O. et al. Quality of life and burden of caregivers of children and adolescents with disabilities. Special Care Dentistry Association and Wiley Periodicals, v. 39, p. 380-388, 2019. DOI: $10.1111 /$ scd. 12400

BEKTAS, I. et al. Symptom frequency in children with congenital heart disease and parental care burden in predicting the quality of life of parents in Turkey. Journal of Pediatric Nursing, v. 53, p. e211-e216, 2020. DOI: 10.1016/j.pedn.2020.04.012

BONIS, S. Stress and parents of children with autism: a review of literature. Issues of Mental Health Nursing, v. 37, n. 3, p. 153-163, 2016. DOI: 10.3109/01612840. 2015.1116030 
CHIAO, C. Y.; WU, H. S.; HSIAO, C. Y. Caregiver burden for informal caregivers of patients with dementia: a systematic review. International Nursing Review, v. 62, n. 3, p. 340-350, 2015. DOI: 10.1111/inr.12194

FÁVERO, M. A. B.; SANTOS, M. A. dos. Autismo infantil e estresse familiar: uma revisão sistemática da literatura. Psicologia: Reflexão e Crítica, v. 18, n. 3, p. 358-369, 2005. DOI: 10.4013/ctc.2012.52.07

GENG, H. M. et al. Prevalence and determinants of depression in caregivers of cancer patients: a systematic review and meta-analysis. Medicine, Baltimore, v. 97, n. 39, p. 1-8, 2018. DOI: 10.1097/MD.0000000000011863

HOEFMAN, R. et al. Autism spectrum disorder and parents' quality of life: application of the CarerQol. Journal of Autism and Development Disorders, v. 44, n. 8, p. 1933-1945, 2014. DOI: 10.1007/s10803-014-2066-1

INSTITUTO BRASILEIRO DE GEOGRAFIA E ESTATÍSTICA. Características gerais dos domicílios e dos moradores: 2012-2019. Pesquisa Nacional por Amostra de Domicílios (Pnad) Contínua, 2019. Disponível em: https://educa.ibge.gov.br/jovens/conheca-o-brasil/populacao/18319-cor-ou-raca.html. Acesso em: 21 jun. 2020.

KOEGEL, R. L. et al. Consistent stress profiles in mothers of children with autism. Journal of Autism Developmental Disorder, v. 22, n. 2, p. 205-216, 1992. DOI: 10.1007/ BF01058151

KOPP, S.; BECKUNG, E.; GILLBERG, C. Developmental coordination disorder and other motor control problems in girls with autism spectrum disorder and/or attention-deficit/hyperactivity disorder. Research Developmental Disability, v. 31, n. 2, p. 350-361, 2010. DOI: $10.1017 /$ s1355617707070270

KOTHARI, R. et al. Gender differences in the relationship between social communication and emotion recognition. Journal of the American Academy of Child and Adolescent Psychiatry, v. 52, n. 11, p. 1148-1157, 2013. DOI: 10.1016/j.jaac.2013.08.006

MAENNER, M. J. et al. Prevalence of autism spectrum disorder among children aged 8 years - autism and developmental disabilities monitoring network, 11 sites, United States, 2016. MMWR Surveillance Summaries, v. 69, n. 4, p. 1-12, 2020. DOI: 10.15585/ mmwr.ss6706a1

MAHENDIRAN, T. et al. Sex differences in social adaptive function in autism spectrum disorder and attention-deficit hyperactivity disorder. Frontiers in Psychiatry, v. 10, n. 607, p. 1-12, 2019. DOI: 10.3389/fpsyt.2019.00607

MARSACK, C. N.; SAMUEL, P. S. Mediating effects of social support on quality of life for parents of adults with autism. Journal of Autism and Developmental Disorders, v. 47, p. 2378-2389, 2017. DOI: 10.1007/s10803-017-3157-6 
MARSACK-TOPOLEWSKI, C. N.; CHURCH, H. L. Impact of caregiver burden on quality of life for parents of adult children with autism spectrum disorder. American Journal on Intellectual and Developmental Disabilities, v. 124, n. 2, p. 145-156, 2019. DOI: 10.1352/1944-7558-124.2.145

MIELE, F. G.; AMATO, C. A. H. Transtorno do espectro autista: qualidade de vida e estresse em cuidadores e/ou familiares - revisão de literatura. Cadernos de Pós-Graduação em Distúrbios do Desenvolvimento, v. 16, n. 2, p. 89-102, 2016. DOI: $10.5935 / 1809-4139.20160010$

MISQUIATTI, A. R. N. et al. Sobrecarga familiar e crianças com transtornos do espectro do autismo: perspectiva dos cuidadores. Revista CEFAC, v. 17, n. 1, p. 192-200, 2015. DOI: $10.1590 / 1982-0216201520413$

SAVIANI-ZEOTI, S.; PETEAN, E. B. L. A qualidade de vida de pessoas com deficiência mental leve. Psicologia: Teoria e Pesquisa, v. 24, n. 3, p. 305-311, 2008. DOI: 10.1590/ S0102-37722008000300006

TEN HOOPEN, L. W. et al. Children with an autism spectrum disorder and their caregivers: capturing health-related and care-related quality of life. Journal of Autism and Developmental Disorders, v. 50, p. 263-277, 2020. DOI: 10.1007/s10803-019-04249-w

THE WHOQOL GROUP. Development of the World Health Organization WHOQOL-BREF quality of life assessment. Psychological Medicine, v. 28, n. 3, p. 551-558, 1998. DOI: $10.1017 / \mathrm{s} 0033291798006667$

VELHOTE, A. B.; BOHOMOL, E.; VELHOTE, M. C. P. Reações do acompanhante diante dos procedimentos pré-operatórios em cirurgia pediátrica ambulatorial. Einstein, São Paulo, v. 14, n. 3, p. 403-407, 2016. DOI: 10.1590/S1679-45082016AO3624

WERLING, D. M.; GESCHWIND, D. H. Sex differences in autism spectrum disorders. Current Opinion in Neurology, v. 26, n. 2, p. 146-153, 2013. DOI: 10.1097/WCO. 0b013e32835ee548 